\title{
Cognitive stimulation therapy improves cognition and quality of life in older people with dementia
}

Spector A, Thorgrimsen L, Woods B, et al. Efficacy of an evidence-based cognitive stimulation therapy programme for people with dementia: randomised controlled trial. Br J Psychiatry 2003;183:248-54.

\section{Does cognitive stimulation therapy improve cognition and quality of life in older people with dementia?}

\section{METHODS}

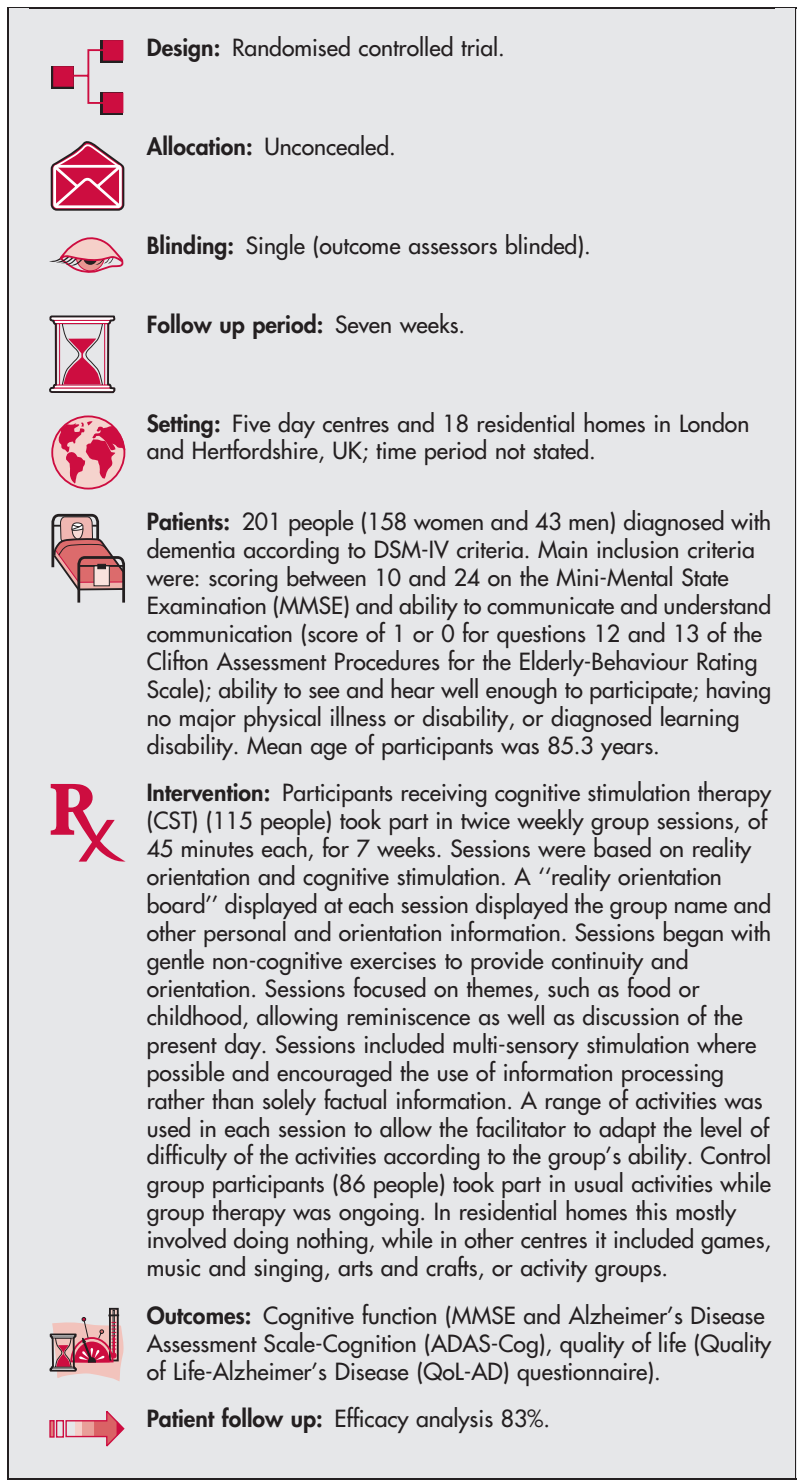

For correspondence: Martin Orrell Department of Psychiatry and Behavioural Sciences, UCL, Wolfson Building, Riding House Street, London, UK; m.orrell@ucl.ac.uk

Sources of funding: The NHS London Regional Office, Research and Development Programme, and Barking, Havering and Brentwood Community NHS Trusts.

\section{MAIN RESULTS}

CST significantly improved cognitive function compared with usual activities (mean change in MMSE score from baseline: +0.9 with CST $v-0.4$ with usual activities, $\mathrm{p}=0.04$; mean change in ADAS-Cog score from baseline: +1.9 with CST $v-0.3$ with usual activities, $\mathrm{p}=0.0 \mathrm{l}$; NNT for 4 point increase in ADAS-Cog score $=6,95 \%$ CI 4 to 17). CST significantly improved quality of life compared with usual activities (mean change in QoL-AD score from baseline: +1.3 with CST $v-0.8$ with usual activities, $\mathrm{p}=0.03$ ).

\section{CONCLUSIONS}

CST significantly improves cognition and quality of life in older people with dementia. Improvements in cognition with CST were comparable for those seen in studies of acetylcholinesterase inhibitors.

\section{NOTES}

Authors note that there was significant variation between centres in improvement in cognitive function (ADAS-Cog score). The control group was smaller than the treatment group as at least 5 people from each centre, often with only 8 or 9 people eligible, were randomised to CST. Randomisation was not performed independently. The control group received heterogeneous "usual activities".

\section{Commentary}

pector et al have provided the first large, multicentred, randomised control trial of cognitive stimulation therapy (CST), a version of a non-medical therapy that has been in existence for almost 50 years. Their design paralleled drug trials in the use of measures of cognition as well as measures of behaviour and quality of life. One important feature of this study was that none of the participants were taking cholinesterase inhibitors at the time of their participation.

This study has a number of important implications for the treatment of dementia and future research. First, there is an alternate, efficacious therapy for individuals who either cannot tolerate cholinesterase inhibitors (or any future medication for dementia) or chose not to take medication. Second, in addition to placebo, CST should be considered as an alternate treatment group in future drug trials. Third, there is a need to study the combined effects of CST with medication. It seems reasonable to assume that CST plus drug therapy would, in the long run, prove to be the most efficacious treatment. Finally, there is a need to extend the time of study. Spector et al ran their trial for only 7 weeks ( 14 sessions held twice weekly) but it would be important to know the effect size over a study period comparable to that used in drug trials (ie 27 to 52 weeks).

From a personal standpoint, this study provides crucial evidence that current programmes in our institution, designed along similar lines, are providing beneficial care to our clients. There are some obvious limitations to the use of the technique as attested to by the inclusion criteria used in the study. Nevertheless, the authors should actively promote the use of such programmes for treatment of dementia. It would be of significant benefit if they could train caregivers and staff of long term care facilities in order that the programmes would become a standard of care for dementia.

Larry Leach, PhD, Psychologist Department of Psychology, Baycrest Centre for Geriatric Care, Toronto, Ontario, Canada 